\title{
The adaptive dynamics of Lotka-Volterra systems with trade-offs
}

\author{
Roger G. Bowers ${ }^{\mathrm{a}}$, Andrew White ${ }^{\mathrm{b}, *}$ \\ ${ }^{a}$ Department of Mathematical Sciences, University of Liverpool, P.O. Box 147, Liverpool L69 3BX, UK \\ ${ }^{\mathrm{b}}$ Department of Mathematics, Heriot-Watt University, Edinburgh EH14 4AS, UK
}

Received 31 May 2001; received in revised form 31 October 2001; accepted 14 November 2001

\begin{abstract}
We analyse the adaptive dynamics of a generalised type of Lotka-Volterra model subject to an explicit trade-off between two parameters. A simple expression for the fitness of a mutant strategy in an environment determined by the established, resident strategy is obtained leading to general results for the position of the evolutionary singular strategy and the associated second-order partial derivatives of the mutant fitness with respect to the mutant and resident strategies. Combinations of these results can be used to determine the evolutionary behaviour of the system. The theory is motivated by an example of prey evolution in a predator-prey system in which results show that only (non-EUS) evolutionary repellor dynamics, where evolution is directed away from a singular strategy, or dynamics where the singular strategy is an evolutionary attractor, are possible. Moreover, the general theory can be used to show that these results are the only possibility for all Lotka-Volterra systems in which aside from the trade-offs all parameters are independent and in which the interaction terms are of quadratic order or less. The applicability of the theory is highlighted by examining the evolution of an intermediate predator in a tri-trophic model. (c) 2002 Elsevier Science Inc. All rights reserved.
\end{abstract}

Keywords: Lotka-Volterra; Adaptive dynamics; Trade-offs; Speciation

\section{Introduction}

The analysis presented in this article depends on two basic ideas. The first of these is that of adaptive dynamics [1-3] - an evolutionary theory which provides a dynamic counterpart to the

\footnotetext{
* Corresponding author. Tel.: +44-131 451 3222; fax: +44-131 4513249.

E-mail address: awhite@ma.hw.ac.uk (A. White).
} 
theory of evolutionarily stable strategies. The second is that of trade-offs between evolving lifehistory constraints $[4,5]$ - due to energetic constraints a gain in one area must be balanced by a loss in another.

The aim of this paper is to initiate the general theory - adaptive dynamics with trade-offs - which results when these two ideas are combined. Since this is a first attempt at such a theory, we set out to provide a baseline which addresses the most fundamental aspects of the behaviour in question. To this end we focus attention on a particular class of generalised Lotka-Volterra models in which life-history parameters appear in a relatively simple way. This provides our baseline - a firm background against which future, more detailed and complicated behaviour can be viewed.

The theory of adaptive dynamics [1-3] arose by closing a critical feedback - that individuals who constitute a population, 'the residents', will necessarily affect the environment they populate. New types of individuals arise at low density from small mutations around the resident strategy and for this mutant to be successful it must initially prosper in the environment determined by the resident. Successful mutants increase in density and begin to shape the environment. In the long term the mutant may coexist with the original resident or oust it to become the new resident itself. The theory imposes a clear separation of the (slow) evolutionary and (fast) population dynamical time scales, that is, mutations occur sufficiently infrequently that the population has reached its attractor before a new mutation occurs. This is a very realistic assumption for almost all evolutionary situations.

Although the theory of adaptive dynamics has only recently been outlined it is at the forefront of theoretical attempts to explain speciation through a process of evolutionary branching. Studies have highlighted various methods by which branching can occur, such as evolving reproductive compatibility or dispersal rates between population types, environmental heterogeneity, or asymmetric competition (see [6,7] and references therein). The theory has been applied successfully to empirical problems relating to the management of evolving fish stocks [8] and the evolution of seed size and germination strategy [9].

The key expression in the analysis of adaptive dynamics is the fitness function of the mutant strategy which is calculated as the per capita growth rate of a mutant strategy, $y$, in an environment determined by the resident population, $x$, and denoted $s_{x}(y)$. If $s_{x}(y)$ is negative the mutant dies out; if $s_{x}(y)$ is positive it will spread. Given that mutations are small, the population will evolve in the direction of the local fitness gradient, $\left[\partial s_{x}(y) / \partial x\right]_{y=x}$, until it reaches the neighbourhood of a 'singular strategy', $x_{s}$, for which the fitness gradient is zero. The behaviour at the singular strategy is determined from combinations of the associated second-order partial derivatives of the fitness function with respect to the mutant and resident strategies and characterised by four properties (Table 1). For instance if $x_{s}$ is an Evolutionary Unbeatable Strategy (EUS) and Convergence Stable (CS), then it is necessarily an evolutionary attractor (called a continuously stable strategy or CSS $[1,3]$ ), if it is not EUS and not CS it is a (non-EUS) evolutionary repellor [1]. The phenomenon of branching occurs when $x_{s}$ is CS but not EUS. Here we evolve towards $x_{s}$ but when close by undergo disruptive selection and two distinct strategies coexist either side of $x_{s}$. (See [1-3] for more details on the theory of adaptive dynamics.)

In this study a mutant is characterised as having a small, discrete difference in some of its parameters (or life history characteristics) compared with those of the resident population. One might expect that a mutation would only affect one parameter, but it is becoming increasingly accepted that a benefit gained in one area of a species life history will trade-off with a cost in 
Table 1

Properties of the singular strategy, $x_{s}$

\begin{tabular}{ll}
\hline Property & Characteristics \\
\hline Evolutionarily unbeatable strategy (EUS) & $B<0$ \\
Convergence stable (CS) & $A-B>0$ \\
Singularity can spread (SPR) & $A>0$ \\
Mutually invadable (MI) & $A+B>0$ \\
$\qquad A=\left.\frac{\partial^{2} s_{x}(y)}{\partial x^{2}}\right|_{y=x=x_{s}}, \quad B=\left.\frac{\partial^{2} s_{x}(y)}{\partial y^{2}}\right|_{y=x=x_{s}}$ & \\
\hline
\end{tabular}

another. Thus, it has been argued using simple game theory that without such trade-offs species variation would not occur, as a single type will always have the highest fitness [4]. Trade-offs have been reported in experimental systems where species strains with higher resistance to disease pay a cost in terms of a reduced rate of disease-free reproduction [5] and an exceptional study by [10] showed that the adaptive behaviour and in particular the occurrence of branching was different for three separate trade-off functions which associate higher intrinsic growth rate with higher transmission of infection in host-parasite systems. Thus, explicit relationships between parameters, or trade-offs, are fundamental in the real world.

In this study we will explicitly link parameter pairs using a functional trade-off. Previous studies using adaptive dynamics $[7,11,12]$ have not focussed on the explicit relationships between parameters but rather linked parameters by a relationship with a trait value. Functional trade-offs make clear the connection between parameters and are a subset of the parameterised relationships (for example the parameterised relationships in Refs. [7,11] could not be represented as functional trade-offs) so it is important to examine the evolutionary consequences of this restriction. We shall derive a baseline theory for the adaptive dynamics of (generalised) Lotka-Volterra systems (of any dimension) with functional trade-offs between parameter pairs. Lotka-Volterra systems have been chosen as it is relatively easy to compute the mutant fitness function and because they represent a very important class of models in biology and ecology $[1,13,14]$. To motivate the general theory we shall first consider an example of prey evolution in a predator-prey system. We shall then develop a general theoretical scheme that can represent multi-strain, multi-species Lotka-Volterra systems (such as the predator-prey system in the example, but also more complicated systems) which include the realistic effect of trade-offs between two parameters. The trade-off assumption allows us to reduce the complexity of this fitness function and general results relating to the position and stability of an evolutionarily singular strategy can be determined. By further assuming that all the parameters other than those connected by the trade-off are independent (and that the interaction terms are of quadratic order or less) it is possible to provide a general classification of the adaptive dynamics. This classification limits the evolutionary behaviour in an important way. In particular, branching points are not possible and this directs attention (see Section 4) to mechanisms which may be necessary for speciation to occur. The applicability of the general theory is highlighted using an example of evolution of an intermediate level predator in a tri-trophic system. 


\section{Example: Prey evolution in a predator-prey system with trade-offs}

To motivate the general theory we will first consider prey evolution in a multi-strain predatorprey system where we trade-off the prey growth rate against the ability of the prey to avoid predation. If $N_{i}$ represents the density of the prey strain $i$ when $i \in A 1$ (the set of all possible prey strains) and the density of predator strain $i$ when $i \in A 2$ (the set of all possible predator strains) then the dynamics satisfy

$$
\begin{aligned}
& \frac{\mathrm{d} N_{i}}{\mathrm{~d} t}=N_{i}\left(a_{i}-\sum_{j \in A 1} b_{i j} N_{j}-\sum_{j \in A 2} c_{i j} N_{j}\right) \quad \text { for } i \in A 1, \\
& \frac{\mathrm{d} N_{i}}{\mathrm{~d} t}=N_{i}\left(-d_{i}+\sum_{j \in A 1} e_{i j} N_{j}\right) \quad \text { for } i \in A 2,
\end{aligned}
$$

where the parameter $a_{i}$ is the intrinsic growth rate of prey strain $i, b_{i j}$ is the rate of competition of prey strain $j$ on prey strain $i, c_{i j}$ is the rate of predation of predator strain $j$ on prey strain $i, d_{i}$ is the death rate of predator strain $i$ and $e_{i j}$ is the rate of conversion of predator attacks on prey strain $j$ into births of predator strain $i$. We wish to consider the invasion of a mutant prey strain $y$ into an established (monomorphic in each species) resident community $X$ consisting of prey strain $x$ and predator strain $z$ (i.e., $X=\{x, z\}$ ). The fitness of the mutant prey type attempting to invade the resident population is denoted $s_{X}(y)$ and, from (1), satisfies

$$
s_{X}(y)=a_{y}-b_{y x} N_{x}^{*}-c_{y z} N_{z}^{*},
$$

where $N_{x}^{*}$ and $N_{z}^{*}$, the equilibrium densities of the resident prey and predator populations respectively, are given by

$$
N_{x}^{*}=\frac{d_{z}}{e_{z x}}, \quad N_{z}^{*}=\frac{e_{z x} a_{x}-b_{x x} d_{z}}{e_{z x} c_{x z}} .
$$

Since the resident population is in equilibrium we know that the fitness of prey strain $x$ in the population $X$, denoted $s_{X}(x)$, is zero. Thus, the following expression holds:

$$
s_{X}(x)=a_{x}-b_{x x} N_{x}^{*}-c_{x z} N_{z}^{*}=0 .
$$

(Note, it is the simultaneous solution of (5) with the parallel result for the predator $\left(s_{X}(z)=0\right)$ which gives the equilibrium densities in (4).) We can combine (3) and (5) to get

$$
s_{X}(y)=\left(a_{y}-a_{x}\right)-\left(b_{y x}-b_{x x}\right) N_{x}^{*}-\left(c_{y z}-c_{x z}\right) N_{z}^{*} .
$$

We now introduce the trade-off by assuming that the mutant and resident parameters are the same except for the two parameters linked by the trade-off function. Therefore

$$
b_{y x}=b_{x x} \quad \text { and } \quad a_{j}=f\left(c_{j z}\right) \text { for } j \in A 1 .
$$

For $f$ to be a trade-off function in (7) we recognise that $f^{\prime}>0$ (i.e., energetic constraints will mean that an increase in growth $(a)$ will be bought at the cost of an increased rate of predation $(c)$ ). Eq. (6) simplifies to

$$
s_{X}(y)=\left(f\left(c_{y z}\right)-f\left(c_{x z}\right)\right)-\left(c_{y z}-c_{x z}\right) N_{z}^{*} .
$$


Since we have freedom to choose a 1-1 relationship between prey strains and parameter values we can identify $c_{x z}$ with $x$ and $c_{y z}$ with $y$. (We can do this because the predator strain $z$ is fixed - it refers to a different species in which the same resident strain affects both mutant and resident prey strains.) We can then write (8) as

$$
s_{X}(y)=(f(y)-f(x))-(y-x) N_{z}^{*},
$$

where we recall that the equilibrium density $N_{z}^{*}$ depends on $x$ but not $y$.

The population will evolve in the direction of its local fitness gradient [1-3] defined as $\partial s_{X}(y) /\left.\partial y\right|_{y=x}$, which from (9) can be expressed as

$$
\left.\frac{\partial s_{X}(y)}{\partial y}\right|_{y=x}=f^{\prime}(y)-N_{z}^{*} .
$$

Hence, from (10), if $f$ is a not a trade-off, the fitness gradient has negative sign and evolution continues until the minimum value of $x$ is reached. In the other case - on which we shall concentrate - the fitness gradient may change sign and here evolution may continue until the minimum or maximum value of $x$ is obtained or until it reaches a neighbourhood of a strategy for which the fitness gradient is zero. Such a strategy is known as evolutionarily singular and occurs when

$$
f^{\prime}(x)=N_{z}^{*}
$$

To determine the properties of the singular strategy requires knowledge of the second-order partial derivatives of $s_{X}(y)$ with respect to mutant and resident strategies at the singular strategy $x_{s}$ [1-3]. It is easy to show that

$$
\begin{aligned}
& \left.\frac{\partial^{2} s_{X}(y)}{\partial x^{2}}\right|_{x=y=x_{s}}=-f^{\prime \prime}\left(x_{s}\right), \\
& \left.\frac{\partial^{2} s_{X}(y)}{\partial y^{2}}\right|_{x=y=x_{s}}=f^{\prime \prime}\left(x_{s}\right) .
\end{aligned}
$$

In terms of Table 1 this means that $A=-B$. Hence, we are exactly on the boundary of the region where there are nearby dimorphisms. Further consideration yields a complete classification of possible singularities. When $f^{\prime \prime}\left(x_{s}\right)<0$ (trade-off convex), the singularity is an EUS, is necessarily CS and the singularity can spread (SPR) so that it is certainly a CSS and therefore an evolutionary attractor. When $f^{\prime \prime}\left(x_{s}\right)>0$ (trade-off concave), the singularity is not EUS, CS or SPR: one thinks of a (non-EUS) evolutionary repellor. (The non-generic case $f^{\prime \prime}\left(x_{s}\right)=0$ is marginal.)

\section{General theory for Lotka-Volterra systems with trade-offs}

To construct a general theory of the role of trade-offs in determining the adaptive dynamics of our generalised Lotka-Volterra systems requires representing such systems by a universal notation. This notation is similar to that outlined in $[1,13]$ and is suitable for representing commonly studied communities which include trade-offs. 
For our multi-species, multi-strain generalised Lotka-Volterra systems the dynamics of a single strain, $i$, in a set $A$ of all possible strains (or strategies), can be represented as

$$
\frac{\mathrm{d} N_{i}}{\mathrm{~d} t}=N_{i}\left(\sum_{\alpha \in I} \sum_{j \in A} z_{i j}^{\alpha} p_{i j}^{\alpha} P^{\alpha}\left(N_{j}\right)\right),
$$

where, $\alpha$ runs over the set $I$ of all possible interaction types for this set of strains, $i \in A, j$ runs over $A$ and this set can be partitioned to correspond to different species, $N_{i}$ is the density of strain $i, P^{\alpha}$ is the functional form of the interaction of type $\alpha$ and $p_{i j}^{\alpha}$ is a parameter, chosen positive, associated with the interaction of type $\alpha$ between strains $i$ and $j$. Interactions can only take place for appropriate pairs of species, which we denote $s 1(\alpha)$ and $s 2(\alpha)$ for interaction $\alpha$ (in the case of intrinsic interactions characterised below they must also be between appropriate pairs of strains) and define the quantity $z_{i j}^{\alpha}$ which represents this restriction as follows:

$$
\begin{aligned}
z_{i j}^{\alpha} & =k_{i j}^{\alpha} w_{i j}^{\alpha}, \\
k_{i j}^{\alpha} & =\delta(s[i], s 1(\alpha)) \delta(s[j], s 2(\alpha)),
\end{aligned}
$$

where $\delta$ is the Kronecker delta and $k_{i j}^{\alpha}$ forces $z_{i j}^{\alpha}$ to be zero except when the species associated with strains $i$ and $j$ (denoted $s[i]$ and $s[j]$, respectively) are the particular species $s 1(\alpha)$ and $s 2(\alpha)$ involved in the interaction $\alpha$. We can now distinguish two different types of interaction (subsets of $I$ ). These have corresponding 'weights' $w_{i j}^{\alpha}$ and associated functional properties ascribed to $P^{\alpha}$.

For (intrinsic or self) 'interactions' between the same strain (and consequently the same species) which contribute to the per capita rate at (14) even at the lowest densities, we take

$$
\alpha \in I_{0}, \quad w_{i j}^{\alpha}=\delta(i, j), \quad P^{\alpha}(N) \rightarrow \pm 1 \quad \text { as } N \rightarrow 0 .
$$

An example of this would be a per capita birth or death rate.

For all other interactions, whether between strains belonging to two distinct species or to distinct strains of the same species, there is no contribution to the above per capita rate at the lowest densities and we take

$$
\alpha \in I_{1}, \quad w_{i j}^{\alpha}=1, \quad P^{\alpha}(N) \rightarrow 0 \quad \text { as } N \rightarrow 0 .
$$

An example of this would be an interspecific or intraspecific competition rate.

The notation developed at (14)-(17) has the great advantage of generality and compactness and we use it as a basis for results applicable to a broad class of Lotka-Volterra systems below. However, for heuristic reasons, it is worth making it more tangible by means of an example. Consider the predator-prey system outlined in (1) and (2) where we additionally label the prey species $s 1$ and the predator species $s 2$.

The system can be represented as a single equation as follows

$$
\frac{\mathrm{d} N_{i}}{\mathrm{~d} t}=N_{i}\left(\sum_{j \in A} a_{i} z_{i j}^{1}-\sum_{j \in A} b_{i j} z_{i j}^{2} N_{j}-\sum_{j \in A} c_{i j} z_{i j}^{3} N_{j}-\sum_{j \in A} d_{i} z_{i j}^{4}+\sum_{j \in A} e_{i j} z_{i j}^{5} N_{j}\right), \quad i \in A,
$$

as the $z_{i j}^{\alpha}$ in this equation ensure that only the appropriate terms are considered for a particular strain of a particular species. Formally, the predator-prey system (1) and (2) can be represented by the general Eq. (14) by making the following identifications. 


$$
\begin{aligned}
& \alpha=1 \in I_{0}, \quad k_{i j}^{1}=\delta(s[i], s 1) \delta(s[j], s 1), \quad w_{i j}^{1}=\delta(i, j), \quad p_{i j}^{1}=a_{i}, \quad P^{1}(N)=1, \\
& \alpha=2 \in I_{1}, \quad k_{i j}^{2}=\delta(s[i], s 1) \delta(s[j], s 1), \quad w_{i j}^{2}=1, \quad p_{i j}^{2}=b_{i j}, \quad P^{2}(N)=-N, \\
& \alpha=3 \in I_{1}, \quad k_{i j}^{3}=\delta(s[i], s 1) \delta(s[j], s 2), \quad w_{i j}^{3}=1, \quad p_{i j}^{3}=c_{i j}, \quad P^{3}(N)=-N, \\
& \alpha=4 \in I_{0}, \quad k_{i j}^{4}=\delta(s[i], s 2) \delta(s[j], s 2), \quad w_{i j}^{4}=\delta(i, j), \quad p_{i j}^{4}=d_{i}, \quad P^{4}(N)=-1, \\
& \alpha=5 \in I_{1}, \quad k_{i j}^{5}=\delta(s[i], s 2) \delta(s[j], s 1), \quad w_{i j}^{5}=1, \quad p_{i j}^{5}=e_{i j}, \quad P^{5}(N)=N .
\end{aligned}
$$

For example, for a predator (species $s 2$ ) strain $i \in A 2$ we have that $s[i]=s 2$ and so $\delta(s[i], s 1)=0$. Thus, $z_{i j}^{1}=z_{i j}^{2}=z_{i j}^{3}=0$ and there will be no contribution from terms in which $\alpha=1,2$ or 3 . For $\alpha=4, z_{i j}^{4}=0$ except when $i=j$, producing the contribution from the intrinsic predator death term. For $\alpha=5, z_{i j}^{5}=1$ when $j \in A 1$ and $z_{i j}^{5}=0$ when $j \in A 2$ producing the contribution from predation on all prey strains. By considering the prey species in a similar way we can check that the general Eq. (14) with identity (19) represents the predator-prey system (1) and (2). We have demonstrated how our notation represents a particular Lotka-Volterra system. What needs stressing now is that it applies with great generality. For example it can represent communities of many species (a three species example is presented later), interaction terms of higher order (i.e., $P^{\alpha}(N)=N^{2}$ ) and systems with parameter dependencies. The general theory which follows therefore applies to a wide range of Lotka-Volterra systems.

\subsection{The fitness function for generalised Lotka-Volterra systems with trade-offs}

This paper is concerned with how explicit functional trade-offs between parameters will affect the adaptive dynamics. Therefore our initial results - which parallel those from (1) to (9) in the above example - use the universal notation introduced above to derive an expression for the fitness of a mutant strain subject to a relation between two interaction parameters linked by a defined trade-off function.

Following the method of [1-3] we assume that the system is constituted of a resident community $X$, a proper subset of $A$, made up of one strain of each species (of which there may be arbitrarily many). The resident population (monomorphic in each species) is taken to be in equilibrium, therefore the fitness of any strain $x$ in the resident population $X\left(\operatorname{denoted} s_{X}(x)\right)$ is zero. Mathematically this means that

$$
s_{X}(x)=\sum_{\alpha \in I} \sum_{j \in X} z_{x j}^{\alpha} p_{x j}^{\alpha} P^{\alpha}\left(N_{j}^{*}\right)=0 .
$$

Now (20) really represents a set of equations, one for each resident strain $x \in X$. Thus the *'s refer to the fact that we assume this set can be solved to give a unique equilibrium density $N_{j}^{*}$ for each strain $j \in X$.

We next fix our attention on a particular species with resident strain $x$ and consider the success of invasion by a mutant strain $y$ (of the same species). The fitness of this strain can be described by

$$
s_{X}(y)=\lim _{N_{y} \rightarrow 0}\left\{\sum_{\alpha \in I} \sum_{j \in X \cup\{y\}} z_{y j}^{\alpha} p_{y j}^{\alpha} P^{\alpha}\left(N_{j}^{*}\right)\right\} .
$$

Here it is convenient to slightly abuse notation and take $N_{y}^{*}=N_{y}$ in the summation. The limit in Eq. (21) arises because the mutant is initially rare and therefore of low density. We can combine (20) and (21) to get 


$$
s_{X}(y)=\lim _{N_{y} \rightarrow 0}\left\{\sum_{\alpha \in I} \sum_{j \in X \cup\{y\}}\left(z_{y j}^{\alpha} p_{y j}^{\alpha}-z_{x j}^{\alpha} p_{x j}^{\alpha}\right) P^{\alpha}\left(N_{j}^{*}\right)\right\} .
$$

In preparation for imposing the trade-off we now restrict our attention to mutations which only affect two interaction types and which involve species represented in $X$. One might imagine that mutations would almost invariably only affect one parameter, but here we recognise that changes in one parameter necessarily have an effect on another characteristic of a species life history (the basis of trade-offs). Thus we assume that $p_{y j}^{\alpha}=p_{x j}^{\alpha}$ for all $\alpha$ except $a, b \in I(a \neq b)$. We also note that $k_{x j}^{\alpha}=k_{y j}^{\alpha}$ since $x$ and $y$ belong to the same species (see (15)). Thus, we find that

$$
s_{X}(y)=\lim _{N_{y} \rightarrow 0}\left\{\sum_{j \in X \cup y}\left(w_{y j}^{a} p_{y j}^{a}-w_{x j}^{a} p_{x j}^{a}\right) k_{x j}^{a} P^{a}\left(N_{j}^{*}\right)+\sum_{j \in X \cup y}\left(w_{y j}^{b} p_{y j}^{b}-w_{x j}^{b} p_{x j}^{b}\right) k_{x j}^{b} P^{b}\left(N_{j}^{*}\right)\right\} .
$$

All other terms $(\alpha \neq a, b)$ cancel since $k_{x j}^{\alpha}=k_{y j}^{\alpha}$ and for $\alpha \in I_{1}$ (see (17)) $w_{y j}^{\alpha}=w_{x j}^{\alpha}=1$ or because for $\alpha \in I_{0}$ (see (16)) only two terms are non-zero and these cancel in summation over $j$ (see the results (27) below). This reduction in the number of terms to two - which parallels the disappearance of the parameter $b$ between (6) and (8) in the example - shows the great advantage of using the form at (22) rather than that at (21).

Now $a$ and $b$ must be interaction types that involve the evolving species $s[x]$ and so, using (15), $\delta(s[x], s 1(a))=\delta(s[x], s 1(b))=1$. Therefore, $k_{x j}^{a}$ and $k_{x j}^{b}$ will only be non-zero when $s[j]$ is equal to $s 2(a)$ and $s 2(b)$, respectively (see (15)). As we are considering a resident population which is monomorphic in each species this non-zero value occurs for unique strains in $X$ which we denote $x_{a}$ and $x_{b}$ for $\alpha=a$ and $b$ respectively. (Without loss of generality we note that it is possible for $x_{a}=x_{b}$.) Thus

$$
s_{X}(y)=\lim _{N_{y} \rightarrow 0}\left\{\sum_{j \in\left\{x_{a}, y\right\}}\left(w_{y j}^{a} p_{y j}^{a}-w_{x j}^{a} p_{x j}^{a}\right) k_{x j}^{a} P^{a}\left(N_{j}^{*}\right)+\sum_{j \in\left\{x_{b}, y\right\}}\left(w_{y j}^{b} p_{y j}^{b}-w_{x j}^{b} p_{x j}^{b}\right) k_{x j}^{b} P^{b}\left(N_{j}^{*}\right)\right\},
$$

where $k_{x x_{a}}^{a}=1$ and $k_{x x_{b}}^{b}=1$ (see (15)). Furthermore, if $a, b \in I_{1}$ then for interspecific interactions $k_{x y}^{a}=k_{x y}^{b}=0$ whilst for intraspecific interactions the limit in (17) is operative. Since additionally the $w_{i j}^{\alpha}=1$ (see (17)) Eq. (24) now becomes

$$
s_{X}(y)=\left(p_{y x_{a}}^{a}-p_{x x_{a}}^{a}\right) P^{a}\left(N_{x_{a}}^{*}\right)+\left(p_{y x_{b}}^{b}-p_{x x_{b}}^{b}\right) P^{b}\left(N_{x_{b}}^{*}\right) .
$$

Furthermore, if, for example, the term labelled by $a$ belongs to $I_{0}$, then using (16) its contribution to $(24)$ is, since $x_{a}=x$,

$$
\sum_{j \in\{x, y\}}\left(\delta(y, j) p_{y j}^{a}-\delta(x, j) p_{x j}^{a}\right) P^{a}\left(N_{j}^{*}\right)
$$

Thus in this case the sum on $j$ contributes two terms which in combination replace the appropriate term in (25) by

$$
\left(p_{y y}^{a}-p_{x x}^{a}\right) P^{a}\left(N_{x}^{*}\right) \text {. }
$$

(Note that the second factor is really a constant, namely \pm 1 .)

We can now introduce trade-offs by recognising that the two remaining parameter types are related by a condition of the form 


$$
p_{j x_{a}}^{a}=f\left(p_{j x_{b}}^{b}\right) .
$$

As promised, we can now express the fitness of the mutant strategy in terms the trade-off function

$$
s_{X}(y)=\left(f\left(p_{y x_{b}}^{b}\right)-f\left(p_{x x_{b}}^{b}\right)\right) P^{a}\left(N_{x_{a}}^{*}\right)+\left(p_{y x_{b}}^{b}-p_{x x_{b}}^{b}\right) P^{b}\left(N_{x_{b}}^{*}\right)
$$

By letting the trade-off be $p_{j j}^{a}=f\left(p_{j x_{b}}^{b}\right)$ it is easy to encompass the case defined by (27). Clearly (29) is a generalisation of (8). It not only encompasses the evolution of different pairs of trade-off parameters but also allows the evolving species to belong to an 'arbitrary' multi-species community (in which the all other species have fixed resident strains). Eq. (29) represents the mutant fitness function for any model that can be represented by (14) and that contains trade-offs between two parameters.

We now invoke a 1:1 correspondence between strains $x$ and $y$ of our evolving species and parameter values $p_{x x_{b}}^{b}$ and $p_{y x_{b}}^{b}$. In the predator-prey example we noted that this identification was allowed because the fixed strain $z$ belonged to a different species to the evolving species. It is also allowed in the general theory if $x_{a}$ and $x_{b}$ are of a different species to that of the resident strain $x$. Additionally, we can allow these strains to be identical with $x$ provided that the parameter is associated with a self-interaction term, so in practice the number of subscripts can be reduced to one (see (27) and for motivation the predator-prey example above). These possibilities cover a large class of interactions for which we will find general results below. (If $x_{a}$ or $x_{b}$ is identical with $x$ and the parameter is not associated with a self-interaction term the general theory will still apply but only in the rather restricted situation in which the mutant parameter is independent of the resident strain.)

By identifying $p_{x x_{b}}^{b}$ with $x$ and $p_{y x_{b}}^{b}$ with y we can write (29) as

$$
s_{X}(y)=(f(y)-f(x)) P^{a}\left(N_{x_{a}}^{*}\right)+(y-x) P^{b}\left(N_{x_{b}}^{*}\right),
$$

which is easy to use particularly if we remember that the densities are those of the resident strains coupled to $x$ and $y$ by the interactions $a$ and $b$. These densities satisfy (20) and hence can depend on $x$ but not $y$.

To distinguish trade-offs from other functions we require that the two terms in (30) have opposite signs. This means that $\operatorname{sign}\left(P^{a}\left(N_{x_{a}}^{*}\right) / P^{b}\left(N_{x_{b}}^{*}\right)\right) f$ is a decreasing function. Since, in applications, $P^{a}\left(N_{x_{a}}^{*}\right)$ and $P^{b}\left(N_{x_{b}}^{*}\right)$ are of fixed sign, we make this a condition and distinguish trade-offs by the requirement that

$$
\operatorname{sign}\left(P^{a}\left(N_{x_{a}}^{*}\right) / P^{b}\left(N_{x_{b}}^{*}\right)\right) f^{\prime}<0 .
$$

\subsection{The adaptive dynamics of general Lotka-Volterra systems}

The theoretical approach of the previous section can be used to represent 'any' Lotka-Volterra system. It will now be used to derive clear-cut and informative general results regarding the evolution of one species in a multi-species resident environment. These results generalise those given near (11)-(13) in the example. We start with the result [1-3] that a population will evolve in the direction of its local fitness gradient which from (30) is

$$
\partial s_{X}(y) /\left.\partial y\right|_{y=x}=f^{\prime}(x) P^{a}\left(N_{x_{a}}^{*}\right)+P^{b}\left(N_{x_{b}}^{*}\right)=P^{b}\left(N_{x_{b}}^{*}\right)\left(\left(P^{a}\left(N_{x_{a}}^{*}\right) / P^{b}\left(N_{x_{b}}^{*}\right)\right) f^{\prime}(x)+1\right) .
$$


This allows us to deduce, from (31), that if $f$ is a not a trade-off, the fitness gradient has fixed sign and evolution continues until the maximum $\left(P^{b}\left(N_{x_{b}}^{*}\right)>0\right)$ or minimum $\left(P^{b}\left(N_{x_{b}}^{*}\right)<0\right)$ value of $x$ is reached. If $f$ is a trade-off then the fitness gradient may change sign and here evolution continues either as above or until it reaches a neighbourhood of the evolutionarily singular strategy. For the generalised Lotka-Volterra systems with trade-offs between two parameters, it is clear (from (32)), that this occurs when

$$
f^{\prime}(x)=-\frac{P^{b}\left(N_{x_{b}}^{*}\right)}{P^{a}\left(N_{x_{a}}^{*}\right)} .
$$

This clear-cut general result indicates that a singular strategy occurs when the slope of the tradeoff function and the above quotient involving at most two population densities at the resident equilibrium are equal. To determine the properties of the singular strategy requires knowledge of the second-order partial derivatives of $s_{X}(y)$ with respect to mutant and resident strategies at the singular strategy $x_{s}[1-3]$. It is easy to show that

$$
\left.\frac{\partial^{2} s_{X}(y)}{\partial y^{2}}\right|_{x=y=x_{s}}=f^{\prime \prime}\left(x_{s}\right) P^{a}\left(N_{x_{a}}^{*}\right) .
$$

Given the sign of $P^{a}\left(N_{x_{a}}^{*}\right)$, whether or not a singular strategy is an EUS is entirely determined by the sign of $f^{\prime \prime}\left(x_{s}\right)$. From the definition in Table 1 it can be seen that if $P^{a}\left(N_{x_{a}}^{*}\right)$ is positive and we consider, for example, a convex trade-off $\left(f^{\prime \prime}\left(x_{s}\right)<0\right)$ then $x_{s}$ is an EUS whereas for a concave trade-off $\left(f^{\prime \prime}\left(x_{s}\right)>0\right) x_{s}$ is not EUS. (If $P^{a}\left(N_{x_{a}}^{*}\right)$ is negative the converse is true.) We can combine these two results in one biological principle which states that $x_{s}$ is an EUS if the trade-off is increasingly marginally costly and is not an EUS if the trade-off is decreasingly marginally costly. To see this it pays to be tangible and consider trade-offs between parameters for which the signs of $P^{a}\left(N_{x_{a}}^{*}\right)$ and $P^{b}\left(N_{x_{b}}^{*}\right)$ differ and so, from (31), $f^{\prime}>0$. Suppose we regard the 'benefit' as the horizontal axis variable $p_{x x_{b}}^{b}$ and the cost as the vertical axis variable $p_{x x_{a}}^{a}=f\left(p_{x x_{b}}^{b}\right)$. Then if $P^{a}\left(N_{x_{a}}^{*}\right)>0$ (and hence $P^{b}\left(N_{x_{b}}^{*}\right)<0$ ), both costs and benefits will increase towards the origin and a convex function will be increasingly marginally costly. Similarly if $P^{a}\left(N_{x_{a}}^{*}\right)<0$ (and hence $P^{b}\left(N_{x_{b}}^{*}\right)>0$ ), both costs and benefits will increase away from the origin and a concave function will be increasingly marginally costly. In cases where $P^{a}\left(N_{x_{a}}^{*}\right)$ and $P^{b}\left(N_{x_{b}}^{*}\right)$ have the same sign costs and benefits increase in 'opposite' directions, conditions on $f^{\prime}$ and $f^{\prime \prime}$ 'flip' but the principle still applies.

To further categorise the evolutionary behaviour around $x_{s}$ we differentiate (30) with respect to $x$ to give

$$
\frac{\partial s_{X}(y)}{\partial x}=(f(y)-f(x)) \frac{\partial P^{a}\left(N_{x_{a}}^{*}\right)}{\partial x}-f^{\prime}(x) P^{a}\left(N_{x_{a}}^{*}\right)+(y-x) \frac{\partial P^{b}\left(N_{x_{b}}^{*}\right)}{\partial x}-P^{b}\left(N_{x_{b}}^{*}\right) .
$$

This evaluates to zero at $x=y=x_{s}$ in agreement with general theory [1-3]. Differentiating a second time with respect to strategy $x$ we get

$$
\begin{aligned}
\frac{\partial^{2} s_{X}(y)}{\partial x^{2}}= & (f(y)-f(x))^{2} \frac{\partial P^{a}\left(N_{x_{a}}^{*}\right)}{\partial x^{2}}-2 f^{\prime}(x) \frac{\partial P^{a}\left(N_{x_{a}}^{*}\right)}{\partial x} \\
& -f^{\prime \prime}(x) P^{a}\left(N_{x_{a}}^{*}\right)+(y-x)^{2} \frac{\partial^{2} P^{b}\left(N_{x_{b}}^{*}\right)}{\partial x^{2}}-2 \frac{\partial P^{b}\left(N_{x_{b}}^{*}\right)}{\partial x} .
\end{aligned}
$$


At the singular strategy this reduces to

$$
\left.\frac{\partial^{2} s_{X}(y)}{\partial x^{2}}\right|_{x=y=x_{s}}=-2 f^{\prime}\left(x_{s}\right) \frac{\partial P^{a}\left(N_{x_{a}}^{*}\right)}{\partial x}-2 \frac{\partial P^{b}\left(N_{x_{b}}^{*}\right)}{\partial x}-f^{\prime \prime}\left(x_{s}\right) P^{a}\left(N_{x_{a}}^{*}\right),
$$

where derivatives are evaluated at $x_{s}$. Eq. (37) is again quite general but it is more difficult to interpret because the derivatives of $P^{a}\left(N_{x_{a}}^{*}\right)$ and $P^{b}\left(N_{x_{b}}^{*}\right)$ are not of fixed sign and magnitude. In some circumstances, however, (37) can be simplified and tractable, general, results for a sub-class of Lotka-Volterra systems can be determined. We take this up immediately in the next paragraph.

\subsection{A general result for Lotka-Volterra systems with independent parameters}

We use the general theory to determine the evolutionary behaviour of a broad sub-class of Lotka-Volterra systems subject to the additional condition that all parameters, except for those linked by the trade-off, are independent. (We also assume that the functional form of the interaction terms is quadratic or less - the $P^{\alpha}(N)$ in (14) are \pm 1 or $\pm N$.) The predator-prey system (1) and (2) is an example of this Lotka-Volterra sub-class. Under this set-up it is possible to show that, for $x=x_{s}, \partial P^{a}\left(N_{x_{a}}^{*}\right) / \partial x=0$ and $\partial P^{b}\left(N_{x_{b}}^{*}\right) / \partial x=0$ (see Appendix A) and therefore (37) becomes

$$
\left.\frac{\partial^{2} s_{X}(y)}{\partial x^{2}}\right|_{x=y=x_{s}}=-f^{\prime \prime}\left(x_{s}\right) P^{a}\left(N_{x_{a}}^{*}\right)
$$

Combining (38) with the general result (34) we have that in terms of Table $1 A=-B$. Thus if $P^{a}\left(N_{x_{a}}^{*}\right)$ is positive and we consider, for example, a convex trade-off $\left(f^{\prime \prime}\left(x_{s}\right)<0\right)$ then $x_{s}$ is an EUS and CS and is therefore an evolutionary attractor (a CSS). For a concave trade-off $\left(f^{\prime \prime}\left(x_{s}\right)>0\right) x_{s}$ is not EUS, not CS and is therefore a (non-EUS) evolutionary repellor. (If $P^{a}\left(N_{x_{a}}^{*}\right)$ is negative the converse is true.) Thus, for a trade-off between parameter pairs in this sub-class of Lotka-Volterra systems the evolving species can only tend towards an evolutionary attracting singular strategy or be repelled from it. Furthermore, it is easy to use the general theory to determine the evolution of any single species under any (relevant) trade-off. Thus, if we had considered predator evolution in the predator-prey example (1) and (2) we would have found that the same evolutionary outcomes (attractor or repellor) were the only possibilities. We further highlight the applicability of the general theory by examining evolution in a tri-trophic system.

\subsection{Evolution in a tri-trophic system}

We consider the following system where $N_{i}$ represent the density of the basal prey if $i \in A 1$, the intermediate predator if $i \in A 2$ and the top predator if $i \in A 3$.

$$
\begin{aligned}
& \frac{\mathrm{d} N_{i}}{\mathrm{~d} t}=N_{i}\left(a_{i}-\sum_{j \in A 1} b_{i j} N_{j}-\sum_{j \in A 2} c_{i j} N_{j}-\sum_{j \in A 3} d_{i j} N_{j}\right) \quad \text { for } i \in A 1, \\
& \frac{\mathrm{d} N_{i}}{\mathrm{~d} t}=N_{i}\left(-e_{i}+\sum_{j \in A 1} g_{i j} N_{j}-\sum_{j \in A 3} h_{i j} N_{j}\right) \text { for } i \in A 2,
\end{aligned}
$$




$$
\frac{\mathrm{d} N_{i}}{\mathrm{~d} t}=N_{i}\left(-k_{i}+\sum_{j \in A 1} l_{i j} N_{j}+\sum_{j \in A 2} m_{i j} N_{j}\right) \text { for } i \in A 3 .
$$

Here the parameter $a_{i}$ is the growth rate of basal prey strain $i, b_{i j}$ is the rate of competition of basal prey strain $j$ on basal prey strain $i, c_{i j}$ and $d_{i j}$ are the rate of predation of intermediate and top predator strain $j$ (respectively) on basal prey strain $i, e_{i}$ is the death rate of intermediate predator strain $i, g_{i j}$ is the rate of conversion of predator attacks on basal prey strain $j$ into births of intermediate predator strain $i, h_{i j}$ is the rate of predation of top predator strain $j$ on intermediate predator strain $i, k_{i}$ is the death rate of top predator strain $i, l_{i j}$ and $m_{i j}$ are the rate of conversion of top predator attacks on basal prey and intermediate predator strain $j$ (respectively) into births of top predator strain $i$.

Let us assume that there is a monomorphic resident environment containing strains $X=\left\{z_{1}, x, z_{2}\right\}$ for the basal prey, intermediate predator and top predator respectively. We wish to examine the evolution of the intermediate predator species under a trade-off between the conversion rate from predation of the intermediate predator on the basal prey $(g)$ and predation rate of the top predator on the intermediate predator $(h)$. To do this we take $g_{j z_{1}}=f\left(h_{j z_{2}}\right)$ or $p_{j z_{1}}^{a}=$ $f\left(p_{j z_{2}}^{b}\right)$ to link with the general theory (28) and note that $P^{a}\left(N_{z_{1}}^{*}\right)=N_{z_{1}}^{*}$ and $P^{b}\left(N_{z_{2}}^{*}\right)=-N_{z_{2}}^{*}$ (see (20)). From (33), the singular strategy, $x_{s}$, occurs when

$$
f^{\prime}(x)=\frac{N_{z_{2}}^{*}}{N_{z_{1}}^{*}} .
$$

From (34)

$$
\left.\frac{\partial^{2} s_{X}(y)}{\partial y^{2}}\right|_{x=y=x_{s}}=f^{\prime \prime}\left(x_{s}\right) N_{z_{1}}^{*}
$$

and from (37) and using the general result for Lotka-Volterra systems with independent parameters (see (38) and the Appendix A) we know that

$$
\left.\frac{\partial^{2} s_{X}(y)}{\partial x^{2}}\right|_{x=y=x_{s}}=-f^{\prime \prime}\left(x_{s}\right) N_{z_{1}}^{*} .
$$

We have used the general theory to show that in terms of Table $1 A=-B$ and therefore the only possible evolutionary outcomes are that the singular strategy is an evolutionary attractor or a (non-EUS) repellor. This three species example highlights the usefulness of the general theory. We could equally have examined evolution of the basal prey or top predator (with trade-offs) with the general theory implying that the same evolutionary outcomes are the only possibility.

\section{Discussion}

We have outlined a general approach for a class of Lotka-Volterra systems which under the assumption of a trade-off between parameters allows the fitness function of an evolving species in a multi-species resident environment to be reduced to a linear combination of the difference in mutant and resident parameter values. The coefficients of this combination are interaction terms 
dependent on the resident population parameters (see (30)). In our study of the adaptive dynamics of such systems we have shown that the position of the singular strategy is determined by the condition that the slope of the trade-off function must be equal to a simple quotient involving at most two population densities evaluated at the resident equilibrium (see (33)). Furthermore, we have determined a general expression that indicates that a singular strategy's EUS status is determined by the curvature of the trade-off function at the singular strategy (see (34)). This can be interpreted biologically by considering trade-offs which are increasingly or decreasingly (marginally) costly. We have also determined an expression for the second-order partial derivative of the mutant fitness with respect to the resident strain of the evolving species (i.e., $\partial^{2} s_{X}(y) / \partial x^{2}$, see (37)). These general results can be used to determine the evolution of a single species against a fixed multi-species environment for Lotka-Volterra systems which include trade-offs.

The general results for $\partial^{2} s_{X}(y) / \partial x^{2}$ obtained in (37) can be simplified to an expression which is directly proportional to the curvature of the trade-off function by considering a sub-class of Lotka-Volterra systems in which all the parameters - except those linked by the trade-off - are independent (and in which there are at most quadratic interactions, see (38)). This sub-class still represents a broad range of biological systems and examples have been given of the evolution of a prey species in a predator-prey system and of an intermediate predator in a tri-trophic model. In general, this sub-class of Lotka-Volterra systems can only exhibit evolutionary attractor (CSS) or (non-EUS) evolutionary repellor dynamics (see Appendix A) regardless of the number of species which make up the resident environment of the evolving species or the (relevant) trade-off parameter pair. Since the Lotka-Volterra framework we have considered represents the underlying structure in many models in ecology and biology [14] the result here has wide ranging consequences. The repellor behaviour occurs when the system is not EUS and not CS. In this scenario the strategy will evolve to either its maximum or minimum value, implying that the parameters are at extremes. The attractor behaviour occurs when the singular strategy is both an EUS and CS. This can be interpreted biologically as requiring an increasingly marginally costly trade-off. Such a mechanism has been invoked as a method by which disease virulence is maintained at intermediate levels [15] and the results here suggests that parallel behaviour may be important in many more situations.

A notable result to emerge from the theory of adaptive dynamics is the possibility of a singular strategy being a branching point. At such points population strategies separate and diverge to form a distinct dimorphic population. This process has been suggested as a possible mechanism for speciation (see [7]). We have shown that for large and commonly used descriptions of LotkaVolterra systems with trade-offs branching is not possible. This is not to say that branching cannot occur in Lotka-Volterra systems but it opens a debate on precisely what mechanisms are required for branching to be present. Certainly a more complicated set-up than that considered in the subclass of Lotka-Volterra models defined in Appendix A of this study is needed.

In previous studies $[7,11]$ branching has been shown to be a feature of Lotka-Volterra systems. So how do these set-ups differ from the examples considered in this study? In [7,11] a multi-strain single-species competition model is examined in which explicit functional trade-offs are not considered and instead parameters are linked to an underlying trait value. Furthermore, the parameters can depend jointly on mutant and resident trait values and therefore the 1:1 identification used in our general theory in (30) would not be appropriate (since $y$ is not independent of $x$ ). This 'new' model set-up which differs in two respects from ours - it is not described in terms of trade-offs 
and does admit 'evolving-resident dependency' of parameters - can display branching points. Moreover, one can prove directly (pers. comm. from a referee) that, whether or not one uses tradeoffs, single species models of the type studied in [7,11], but in which the parameters are independent of the resident, can display only evolutionary attractor (CSS) or (non-EUS) evolutionary repellor dynamics (branching is not possible). Thus it is the dependence on the resident strain of the evolving species that underlies the branching seen in Refs. [7,11]. However, we should not look to this dependence as a necessary mechanism in our study of the effects of trade-offs. First the general proof mentioned above fails for multi-species systems and, second, the 1:1 identification used in (30) is appropriate when parameters link to a non-evolving species. For evolution of one species against a fixed multi-species resident environment, as detailed in the examples and represented by our general theory, the result that branching does not occur explicitly arises through the simplification and assumptions associated with the sub-class of Lotka-Volterra systems and the trade-off (see Appendix A). The mutant parameters in these systems are independent of the resident strain of the evolving species and the inclusion of trade-offs is vital. So what feature of the sub-class is responsible for our negative finding - do we have any clues to the mechanisms within the broader class of models allowed until (37) which may lead to branching? One clue is provided by the study [10], which examines the evolution of resistance to disease in a Lotka-Volterra system in which the mutant parameters are independent of the resident strain and in which parameter pairs are linked by a trade-offs. In this model branching can occur. Moreover, the simplification between (37) and (38) does not occur - there is parameter interdependence additional to that described by the tradeoff. So branching can occur in systems represented by (14) in which the mutant parameters are independent of the resident strain (i.e., the resident effects the mutants fitness through its population density only) if the restriction that the parameters are independent of each other is lifted. A general investigation of this point will form the subject of future research.

To summarise, we have produced general results that provide insight into the evolutionary behaviour of Lotka-Volterra systems with trade-offs. We have shown that in a sub-class of Lotka-Volterra systems in which all the parameters (except those linked by the trade-off) are independent (and in which the interactions are at most quadratic) evolutionary branching is not possible. Branching may be a feature of Lotka-Volterra systems if the parameters depend jointly on resident and mutant strain (trait) values and/or the parameters are not independent of each other.

\section{Appendix A}

Consider a model represented by Eq. (14) which consists of $n$ species with independent parameters (aside from the trade-off) and at most quadratic interaction terms $\left(\alpha \in I_{0}, I_{1}\right)$. Since we know the resident population is in equilibrium we can write (20) as

$$
A N^{*}=-C
$$

where $A(n \times n)$ and $C(n \times 1)$ are defined by

$$
A_{i j}=\sum_{\alpha \in I_{1}} z_{i j}^{\alpha} p_{i j}^{\alpha} \operatorname{sign}\left(P^{\alpha}\left(N_{j}^{*}\right)\right), \quad C_{i}=\sum_{\alpha \in I_{0}} z_{i j}^{\alpha} p_{i j}^{\alpha} \operatorname{sign}\left(P^{\alpha}\left(N_{j}^{*}\right)\right),
$$


and $N^{*}$ is a column vector with $n$ entries representing the equilibrium densities for the $n$-species. Furthermore, we invoke a trade-off between two of the parameters associated with (say) the quadratic terms involving the species with resident strain $x$, so that say $p_{x x_{a}}^{a}=f\left(p_{x x_{b}}^{b}\right)$ and hence of necessity $z_{x x_{a}}^{a}=z_{x x_{b}}^{b}=1$. Differentiating with respect to $p_{x x_{b}}^{b}$ (or $x$ in general theory) we get

$$
A \mathrm{~d} N^{*}+\mathrm{d} A N^{*}=0
$$

and we can multiply through by $A^{-1}$ (the matrix inverse of $A$ which exists if the equilibrium exists) to get

$$
\mathrm{d} N^{*}+A^{-1} \mathrm{~d} A N^{*}=0 .
$$

Since all the parameters are independent $\mathrm{d} A$ is a matrix of zeros except for a $\operatorname{sign}\left(P^{b}\left(N_{x_{b}}^{*}\right)\right)$ in the 'position' of $p_{x x_{b}}^{b}$ and a $\operatorname{sign}\left(P^{a}\left(N_{x_{a}}^{*}\right)\right) f^{\prime}\left(p_{x x_{b}}^{b}\right)$ in the 'position' of $f\left(p_{x x_{b}}^{b}\right)$. Hence $\mathrm{d} A N^{*}$ is a column vector of zeros except for one element

$$
\operatorname{sign}\left(P^{a}\left(N_{x_{a}}^{*}\right)\right) f^{\prime}\left(p_{x x_{b}}^{b}\right) N_{x_{a}}^{*}+\operatorname{sign}\left(P^{b}\left(N_{x_{b}}^{*}\right)\right) N_{x_{b}}^{*},
$$

which is exactly equivalent to $f^{\prime}(x) P^{a}\left(N_{x_{a}}^{*}\right)+P^{b}\left(N_{x_{b}}^{*}\right)$ which is zero at a singular point by (33). Therefore $\mathrm{d} N^{*}=0$ which implies that the derivatives of $P^{a}\left(N_{x_{a}}^{*}\right)$ and $P^{b}\left(N_{x_{b}}^{*}\right)$ in Eq. (37) are zero and the result in the main text is verified. It is straightforward to show that this method also applies to trade-offs between other parameter types.

\section{References}

[1] J.A.J. Metz, S.A.H. Geritz, G. Meszena, F.J.A. Jacobs, J.S. Van Heerwaarden, Adaptive dynamics: a geometrical study of the consequences of nearly faithful reproduction, in: S.J. Van Strien, S.M. Verduyn Lunel (Eds.), Stochastic and Spatial Structures of Dynamical Systems, Elsevier, North-Holland, Amsterdam, 1996, p. 183.

[2] S.A.H. Geritz, J.A.J. Metz, E. Kidsi, G. Meszena, Dynamics of adaptation and evolutionary branching, Phys. Rev. Lett. 78 (1997) 2024.

[3] S.A.H. Geritz, E. Kidsi, G. Meszena, J.A.J. Metz, Evolutionary singular strategies and the adaptive growth and branching of the evolutionary tree, Evol. Ecol. 12 (1998) 35.

[4] S.C. Stearns, The Evolution of Life Histories, Oxford University, Oxford, 1992.

[5] M. Boots, M. Begon, Trade-offs with resistance to a granulosis virus in the Indian meal moth examined by a laboratory evolution experiment, Fun. Ecol. 7 (1993) 528.

[6] U. Dieckmann, Can adaptive dynamics invade, Trends Evol. Ecol. 12 (1997) 128.

[7] M. Doebeli, U. Dieckmann, Evolutionary branching and sympatric speciation caused by different types of ecological interactions, Am. Nat. 156 (2000) S77.

[8] M. Heino, Management of evolving fish stocks, Can. J. Fish. Aq. Sci. 55 (1998) 1971.

[9] S.A.H. Geritz, E. Van der Meijden, J.A.J. Metz, Evolutionary dynamics of seed size and seedling competitive ability, Theor. Pop. Biol. 55 (1999) 324.

[10] M. Boots, Y. Haraguchi, The evolution of costly resistance in host-parasite systems, Am. Nat. 153 (1999) 359.

[11] E. Kidsi, Evolutionary branching under asymmetric competition, J. Theoret. Biol. 197 (1999) 149.

[12] P. Marrow, U. Dieckmann, R. Law, Evolutionary dynamics of predator-prey systems: an ecological perspective, J. Math. Biol. 34 (1996) 556.

[13] D.A. Rand, H.B. Wilson, J.M. McGlade, Dynamics and evolution: evolutionary stable attractors invasion exponents and phenotype dynamics, Philos. Trans. R. Soc. Lond. B 343 (1994) 261.

[14] J.D. Murray, Mathematical Biology, Springer, Berlin, 1993.

[15] R.M. May, R.M. Anderson, Epidemiology and genetics in the coevolution of parasites and hosts, Proc. R. Soc. Lond. B 219 (1983) 281. 